\title{
The Design and Implementation of Virtual Roaming in Yunnan Diqing Tibetan traditional Villages
}

\author{
Lucheng Cao, Wu Xu, Ke Li, Chunjie Jin, Ying Su, and Jin He* \\ School of Electronic and Information Engineering, Yunnan Minzu University, Kunming, China
}

\begin{abstract}
Traditional residence is the continuation of intangible cultural heritage and the primitive soil for development. At present, the protection and inheritance of traditional villages have been impacted by the process of modernization, and the phenomenon of assimilation is very serious. This article takes the above questions as the breakthrough point, and then analyzes why and how to use virtual reality technology to better solve the above problems, and take the Yunnan Diqing Tibetan traditional dwellings as the specific example to explore. First, using VR technology, with real images and sound, the paper simulate a near real virtual world. Secondly, we collect a large amount of real image information, and make the visualization model of building by using 3DMAX software platform, UV Mapping and Rendering optimization. Finally, the Vizard virtual reality development platform was used to establish the roaming system and realize the virtual interaction. The roaming system was posted online so that overcome the disadvantages of not intuitive and low capability of interaction, and these new ideas can give a whole new meaning in the protection projects of the cultural relic buildings. At the same time, visitors could enjoy the "Dian-style" architectural style and cultural connotation of dwelling house in Diqing Yunnan.
\end{abstract}

\section{Introduction}

With urbanization and development of the new countryside are proceeding rapidly, and traditional village of unified mode, this caused the emergence of homogeneity in villages, so that many ancient village assimilation phenomenon is very serious, particularly in Tibetan traditional villages in Shangri-La area of Yunnan Province. The architectural structure and layout of the Tibetan folk dwellings in Yunnan have changed considerably. The steel reinforced concrete and brick and tile has replaced the traditional timberwork structure, so that lost its original national characteristics and historical features .On November 1, 2016, experts and scholars from the first "Shangri-La for World" Rural Tourism Development Forum and "World Village in Yunnan" forum came to Wheelun in Shambhala to sign the "2016 Yunnan Traditional Ancient Village Protection and Development Shangri-La Initiative ", in order to protect Diqing Shangri-La Tibetan traditional village. In addition, the traditional residence has its particularity, it is the original soil of the existence and development of the intangible cultural heritage. Many intangible cultural heritages also disappear with the disappearance of residential houses. It can be said that the protection of a few traditional houses also protects the intangible cultural heritage. Therefore, the protection of national traditional houses has been urgent [1].

In recent years, with the rapid development of virtual reality technology, the combination of VR and network technology has brought the exhibition and protection of cultural relics to a brand-new stage. The virtual reality technology through acquiring image data to build 3D model library of traditional residential buildings, which preserved the original architectural style and spatial relations and a series of important data, moreover can achieve high precision of traditional houses, and permanent protection. It also can unify and integrate cultural relics resources through the Internet, whom to use the VR technology to display cultural relics more comprehensively, vividly and realistically through, in addition, cultural relics can get away from the fence of time and space, so that resources can be shared, and truly become a cultural heritage that all mankind can "own" [2].The use of virtual reality technology will promote the cultural industry to enter the information age more quickly and realize the modernization of cultural relics display and protection. This is also a strategic measure of social and cultural significance in the digital age.

\section{Design of Virtual Roaming System}

\subsection{Construction of 3D Scene Model Library for Tibetan Dwellings}

In the development of virtual reality roaming system, it is necessary to collect a large number of three views, architectural CAD drawings, and a large number of actual images of Tibetan houses in the field to build its three-dimensional model. Furthermore, the 3D model is the basis of the whole roaming system, and the quality of

\footnotetext{
* Corresponding author: hjmwh@sina.com
} 
the model directly affects the running effect and the fidelity of the scene. The system uses 3DMAX software to build village spatial model, the software with powerful function, low price and relatively low requirement for hardware system, and has the advantages of simple operation and so on, which greatly reduces the threshold of use.

Because of the large amount of modeling, according to the layout of traditional residential buildings and the characteristics of the surrounding environment, this paper classifies the building and its internal layout as the main modeling object. The surrounding landscape such as trees, grasslands, hillsides and so on are regarded as secondary modeling objects, which can be imported from the model base, in a word, which can not only guarantee the quality of the main modeling objects, but also save a lot of time to finish the post-rendering.

The 3D model of Tibetan traditional residence and internal layout can be built by 3DMAX. In the process of constructing the model, we must pay attention to restoration of Tibetan blockhouse which includes carving, painting, coating and column beams, purlins, rafters and so on. Through modeling, making UV mapping, the basic model library of Tibetan houses has been completed. Figure 1 shows the hierarchical structure of the virtual scene of folk house [3].

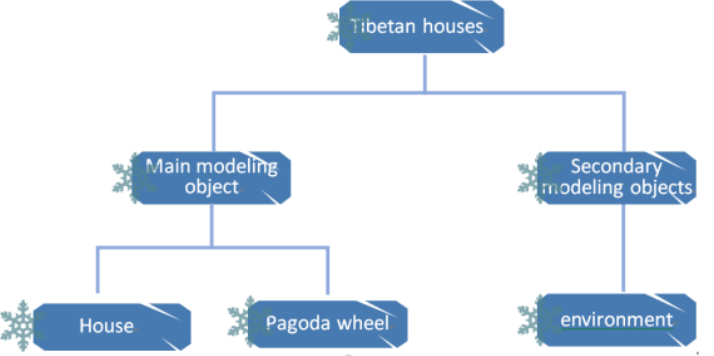

Fig. 1. Hierarchical Structure Diagram of Tibetan Residence Modeling

\subsection{Rendering and Export of Models}

The model rendering also uses 3DMAX software to set the lighting of the scene, thus forming the model rendering map, using the lighting, shadow effect to make the model more realistic, After rendering is complete, we need to install the plug-in OSGexplorer.exe, which can export OSGB file formats that can be recognized by Vizard. After the OSGB tilting photography format is exported, it is also convenient to follow up the operation processing on the Vizard virtual reality engine. In order to ensure that the Vizard can recognize all the information of the model, in this process, we should pay attention to the setting of the parameters when exporting. Since the subsequent virtual interaction is carried out on the virtual reality development platform, Vizard opened up the model built in 3DMAX directly and the modeling results are observed. Figure 2 shows the overall effect of Tibetan houses. Figure 3, Figure4 is part of the effect diagram, from which we can see all the information that Vizard can recognize the model. Accordingly, the virtual scene modeling is successful.
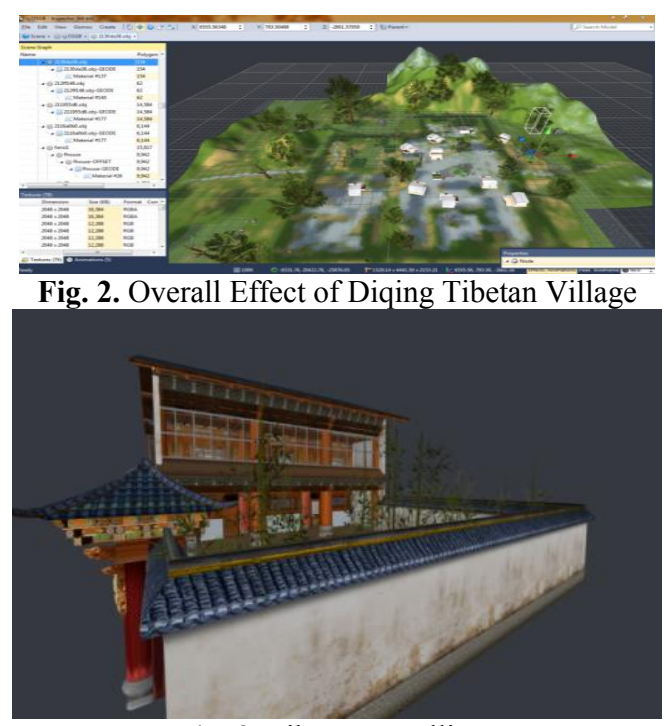

Fig. 3. Tibetan Dwellings

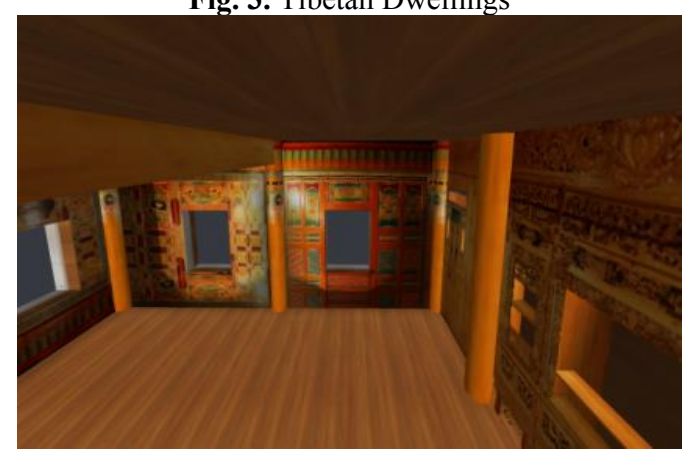

Fig. 4. Interior Painting and Engraving of Houses

\section{Realization of virtual roaming system}

\section{1. the design process of the roaming system}

The design of traditional Tibetan village roaming system can be divided into two processes: one is to construct 3D virtual scene of Tibetan traditional residence, the other is to realize virtual interaction. The main software used in this paper is $3 \mathrm{D}$ modeling software and virtual reality development platform software. According to the characteristics of this paper, and through comparative analysis, we select 3D Max and Vizard as main software.The specific process is shown in Figure 5.

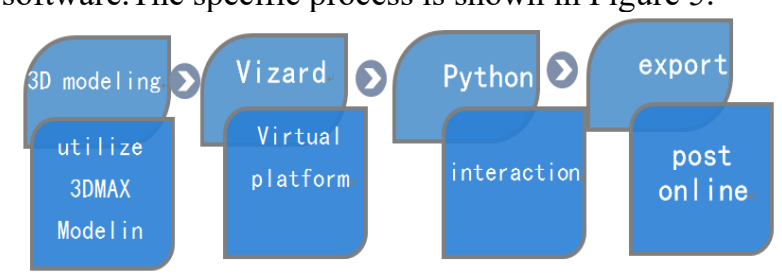

Fig. 5. Design Flowchart of Roaming System

\subsection{Loading 3D Virtual Scene}

The development of virtual roaming system of Tibetan village can not run without a feature-rich development platform, this paper adopts Vizard as the development platform. Compared to other virtual reality development platform, Vizard software provides abundant function modules, users can quickly develop a prototype system 
used in various occasions, moreover, it more easily to get started, and the programming is simple, the function is strong at the same time. The Vizard environment can use the Python language to load the successful 3D virtual model built in the 3DMAX. After loading, users can see the loading scene in the preview window. The Python statement to load the OSGB file is: $\mathrm{cj}=$ viz.addn.cj1.osgb. The basic process of loading a three-dimensional scene is shown in flowchart 6 .

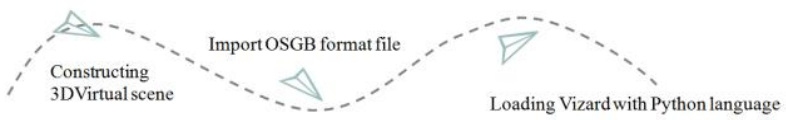

Fig. 6. Loading 3D Scene Flow Chart

\subsection{Interaction of 3D Scenes}

The realization of $3 \mathrm{D}$ scene interaction is the most important part of the development of roaming system, which is mainly divided into two parts: UI option interface and code design.

\subsubsection{UI Option Interface}

UI options interface plays an important role in the interaction of 3D scene, because a simple and fullfeatured interactive page will directly affect the visitor's experience, so the design of the option interface should be fully functional and easy to operate. In this paper, the option interface is divided into five main parts, respectively is general interface, residential interface 1 , residential subinterface 1 , residential interface 2 and residential subinterface 2, to guide the visitor to enter roaming system. options interface hierarchy diagram as shown in figure 7.

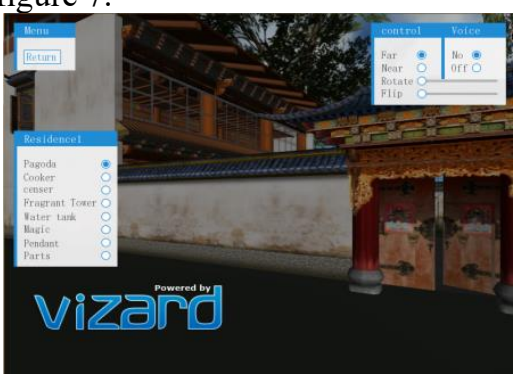

(a)

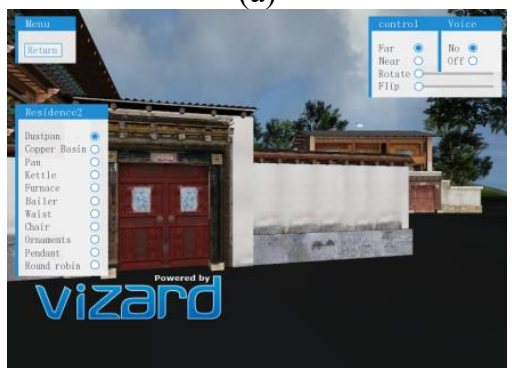

(b)

Fig. 7. Options Interface Hierarchy Diagram

\subsubsection{Coding Design}

The coding design can realize all the functions of the Tibetan residence roaming system, and make the roaming system have virtual interaction, and it is also an indispensable part in human-computer interaction. First of all, to determine the design goal, this paper designed a roaming system which can realize omnidirectional free roaming without dead-angle, and it can be controlled by the mouse angle at the same time. In order to increase the virtual interactive immersion of the roaming system, the audio editing software of Balabolka can be used to edit the sound, so that the audience can listen to the concrete explanation while the audience can enjoy it, so as to realize the function of the voice guide. Meanwhile, we can use Vizard through the Python language to write the content of the scene and achieve audio call [4].

The coding design process is as follows:

1)First, use Vizard to create a new Vizard file and name it as Tibetan dwellings.

2)Second, create a new blank scene, add the scene model (sky) to the blank scene, and adjust the background settings accordingly.

3)Third, establish UI option interface for scene, create UI material, and make main interface for interaction.

4)Then, load the establishing OSGB model, add the corresponding script, and build for the objects that need to interact by setting up the function. Finally realize the interaction of 3D scene, and call the audio at the same time.

5)Finally, test and compile run.

After the coding design is completed, the code is compiled and run successfully, the effect diagram is shown in Figure 8, Figure 9 [5].

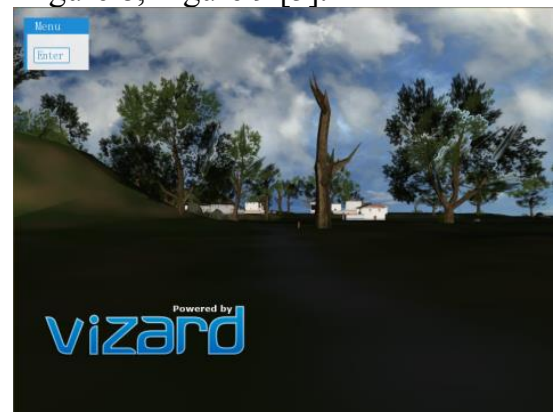

(a)

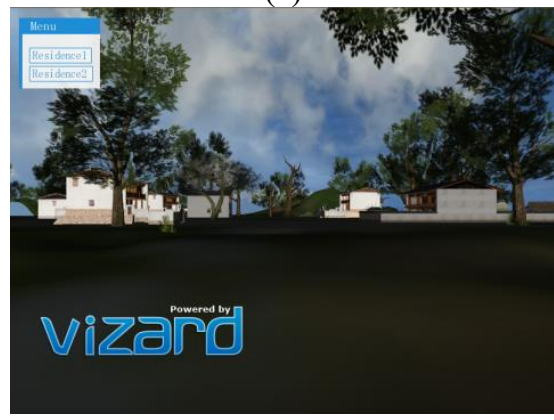

(b)

Fig. 8. Start Interface of Tibetan Dwelling Roaming System 


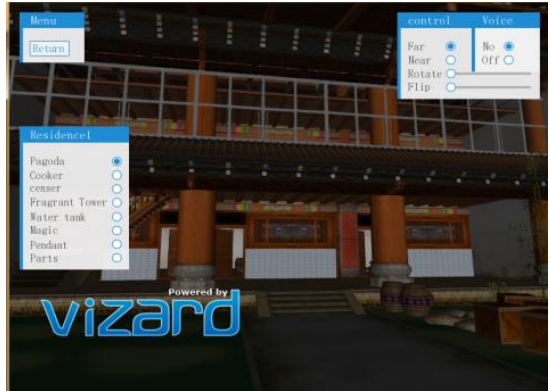

(a)

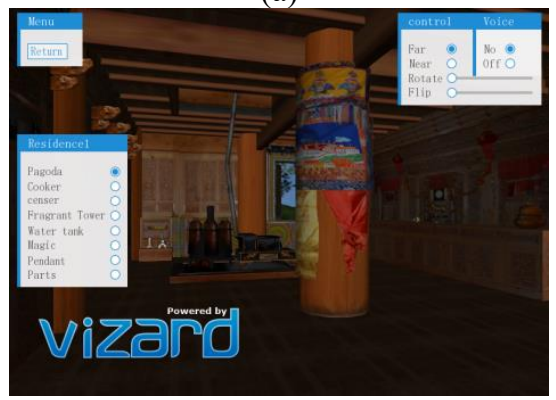

(b)

Fig. 9. Tibetan Dwellings Part1 Showing the Effect Some of the key codes used are:

link $=$

viz.link(groupcm,viz.MainView,srcFlag=viz.ABS_GLO BAL)

$\operatorname{mj} 1=$

modePanel.addItem(viz.addButtonLabel(Tibetan dwelling1'));

$\mathrm{mj} 2=$

modePanel.addItem(viz.addButtonLabel(Tibetan dwelling2'));

vizact.onbuttondown(mj1,CMStart,'cm1');

vizact.onbuttondown(mj2,CMStart,'cm2');

diaoz_mode.endAction()

diaoz_ch.setPosition $(00,00,00)$

diaoz_mode.setAxisAngle([0.0, 0.0, 1.0, 0.0])

shipin_mode.endAction()

shipin_ch.setPosition $(00,00,00)$

shipin_mode.setAxisAngle $([0.0,0.0,1.0,0.0])$

yizi_mode.endAction()

yizi_ch.setPosition $(0,0,0)$

yizi_mode.setAxisAngle([-0.0, 1.0, 0.0, 90.00000762939453])

\subsubsection{Immersive Roaming}

Vizard has strong device compatibility, supports helmet display and many other external input devices, so the use of stereoscopic head display device can achieve immersive roaming effect.This thesis has set Vizard in the code for full-screen display effect, specify the relevant helmet model and add tracking code in the software to track the helmet with the tracking code can achieve virtual roaming. The user can see a full view of the virtual village, and when you walk, you will see the picture will also move, so that the user is out of the real world, completely immersed in the virtual Tibetan dwelling world [6].

\subsubsection{Promotion of Virtual Roaming System}

Vizard can automatically use script the program to generate exe executable file, which means that it can roam on any computer, which provides a prerequisite for the promotion of roaming system. Therefore, this roaming system can post to the Internet, establish an independent website, and build a digital museum, meanwhile, realize the sharing of cultural resources, so that it let more people understand the Tibetan culture, and also help to protect and inherit the regional Tibetan dwelling culture.

\section{Conclusion}

This paper aims at the problem that the traditional villages of ethnic minorities are affected by urbanization and modernization, but lose their original national characteristics and historical heritage. This paper takes the Tibetan dwellings in Diqing Shangri-La, Yunnan Province as an example, puts forward the method of establishing virtual roaming system to protect traditional villages by using the VR technology, so that more people can view the architectural details and architectural characteristics of Tibetan houses at close range. And through the Internet, more people can cross the time and space constraints, enjoy Diqing Shangri-La Tibetan residence with "Dian-style" style anytime and anywhere, so that "cultural relics" really become the wealth of all mankind. The roaming system designed in this paper also provides a way of thinking for the digital protection of other endangered villages. At the same time, this paper also has some shortcomings, such as, due to time constraints, and the carvings of the dwellings are very complicated, the roaming system is still not able to fully depict the real delicate degree of them. But we still hope the research work can provide idea for the protection of cultural relics and contribute to the protection and inheritance of ancient buildings in China.

\section{Acknowledgment}

This research is supported by The National Natural Science Foundation of China: "contact + non-contact" mixed interaction interface design strategy and its key human factors problems (Grant: 61741206)

\section{References}

1. Zheng Xin. Study on the protection of traditional villages [D] .Beijing Construction University, 2014

2. Ren Ning-ning, Zhang Wei, Wu Hong-chuan, et al.Analysis of new forms of network display under $3 \mathrm{D}$ Internet $[\mathrm{J}]$.computer knowledge and Technology, 31(2012)

3. Pan Xiuqiang.Design and implementation of virtual roaming system for ancient villages in Nanxi River based on OSG [J] .Journal of Zhejiang Institute of Technology and Industry, 15(2016)

4. Lin Chen, Lin xiaobin. Modeling of $3 d$ virtual scene and its application in Vizard environment [J]. Journal of minjiang college, 36(2015) 
5. Su Yang. Design and implementation of virtual tour of wuyuan huizhou architecture in jiangxi [D]. Beijing university of technology,2014

6. Zhao Wei, Xie Xiaofang.immersion roaming device based on Free Walking [J] .computer Engineering, 35(2009) 